\title{
Phenotypic Characterization and Deoxyribonucleic Acid Homologies of the "Pseudomonas alcaligenes" Group
}

\author{
E. RALSTON-BARRETT, ${ }^{1}$ N. J. PALLERONI, AND M. DOUDOROFF ${ }^{2}$ \\ Department of Bacteriology and Immunology, University of California, Berkeley, California 94720
}

\begin{abstract}
Eighteen strains of bacteria assigned to the "Pseudomonas alcaligenes" group were characterized. On the basis of phenotypic studies and deoxyribonucleic acid hybridization data, the strains could be accommodated in the two previously named species of the group, $P$. alcaligenes and $P$. pseudoalcaligenes. Both species were shown to be closely related to $P$. mendocina and $P$. stutzeri and more distantly related to the fluorescent species of the same genus.
\end{abstract}

Stanier et al. (11), in a taxonomic survey of the aerobic pseudomonads, described an "alcaligenes" group consisting of the species $P$. alcaligenes and $P$. pseudoalcaligenes. These species were characterized, like the two species of the "acidovorans" group, by the inability to use sugars (with the exception of fructose) as carbon sources; they differed from $P$. acidovorans and $P$. testosteroni primarily in their flagellation (the cells are monotrichous), the ability to grow at $41^{\circ} \mathrm{C}$, and in their more restricted nutritional spectrum. The separation of the seven "alcaligenes"-group strains into the two species was based on the greater nutritional versatility (11) and lower guanine plus cytosine $(G+C)$ contents of the deoxyribonucleic acids (DNA) (3) of the six $P$. pseudoalicaligenes strains as compared to the single $P$. alcaligenes strain. Although there was considerable phenotypic heterogeneity within the group, the number of strains studied was too small for further subdivision.

In an attempt to formulate a better characterization of the "alicaligenes" group, we examined 52 isolates sent to this laboratory under the tentative designation $P$. alicaligenes and $P$. pseudoalcaligenes. Only 11 of these could be identified as strains of the "alcaligenes" group (9; E. Ralston, Ph.D. thesis, Univ. of California, Berkeley, Calif., 1972). We have characterized these latter isolates phenotypically and have performed DNA-DNA hybridization experiments with the entire collection of 18 "alcaligenes"-group strains to determine the relationship between them and other members of the genus Pseudomonas.

\section{MATERIALS AND METHODS}

Bacterial strains. Strains previously assigned to the "P. alcaligenes" group are designated as by

'Present address: Department of Nutrition, University of California, Berkeley, CA 94720.

${ }^{2}$ Deceased 4 April 1975.
Stanier et al. (11). Strains 440 and 441 were received from M. Véron, Pasteur Institute, as 63AD and 63AS, respectively. All other $P$. alcaligenes and $P$. pseudoalcaligenes strains were received from M. J. Pickett, Department of Bacteriology, University of California, Los Angeles, Calif. Strain K-384 was isolated as an equipment contaminant. Strains K379 and K-477 were isolated from patients who had undergone tracheotomies. The original sources of $\mathrm{K}$ $311, \mathrm{~K}-401, \mathrm{~K} 504, \mathrm{~K}-532, \mathrm{~K}-546$, and $\mathrm{K}-614$ are not recorded. Strains of all other Pseudomonas species reported here are designated as in our previous publications $(1,2,4,7,9)$.

Methods. Most of the methods used for the phenotypic characterization of the strains are described by Stanier et al. (11) and Palleroni and Doudoroff (6). The phenotypic similarity coefficients (10) for positive characters only $\left(\mathrm{S}_{\mathrm{J}}\right)$ and for positive and negative matches $\left(\mathrm{S}_{\mathrm{SM}}\right)$ were calculated by using 169 morphological, nutritional, and biochemical characters that we had found useful for differentiating between Pseudomonas species. DNA-DNA hybridizations were performed by the competition and direct-binding techniques as described by Ballard et al. (1) and by Palleroni and Doudoroff (5). Two annealing temperatures were used in the competition experiments: the "optimal" one of $25^{\circ} \mathrm{C}$ below the melting point $\left(T_{m}\right)$ of the immobilized DNA and $80^{\circ} \mathrm{C}$ as a more "stringent" test of competitive binding.

\section{RESULTS AND DISCUSSION}

Of the 11 strains newly assigned to the "alcaligenes" group, two have been designated as $P$. alcaligenes (K-384 and K-477) and nine have been designated as $P$. pseudoalcaligenes $(440$, 441, K-311, K-379, K-401, K-504, K-532, K-546, and $\mathrm{K}-614)$ on the basis of their resemblance, respectively, to $P$. alcaligenes strain 142 and the $\operatorname{six} P$. pseudoalcaligenes strains $(63,65,66$, 297, 299, and 417) described by Stanier et al. (11). The assignment of strain K-477 is tentative; although it resembled the other two $P$. alcaligenes strains more than it resembled any strains of $P$. pseudoalcaligenes, it differed from the former in many respects, and it may even- 
tually be shown to represent a separate species within the group.

Tables 1,2 , and 3 present the phenotypic characteristics of the newly identified strains along with the data for the original seven "alcaligenes"-group strains previously characterized. The results show that there is considerable phenotypic variation among the strains examined, even with respect to the accumulation of poly$\beta$-hydroxybutyrate as cellular reserve and the possession of a constitutive arginine dihydrolase, two traits previously shown to be of taxonomic significance within the genus Pseudomonas. It can also be seen that the range of utiliza- ble substrates for this group is rather restricted and that although $P$. alcaligenes strains are in general more restricted than are strains of $P$. pseudoalcaligenes, the two species are not easily distinguished on phenotypic grounds. Only nine of the 134 substrates tested supported the growth of all strains; the number of substrates used by any one strain ranged from 24 to 31 for $P$. alcaligenes and from 28 to 45 for $P$. pseudoalcaligenes. Fructose was the only substrate used by all strains of $P$. pseudoalcaligenes and none of the $P$. alcaligenes strains, although five other substrates not used by any of the latter (glycerate, $\beta$-hydroxybutyrate, mesaconate,

TABLE 1. Phenotypic characters of $P$. alcaligenes and $P$. pseudoalcaligenes

\begin{tabular}{|c|c|c|}
\hline \multirow[b]{2}{*}{ Character } & \multicolumn{2}{|r|}{ No. $(\%)$ of positive strains } \\
\hline & $\begin{array}{l}P . \text { alcaligenes } \\
{[N=3]}\end{array}$ & $\begin{array}{c}P . \text { pseudoalcaligenes } \\
{[N=15]}\end{array}$ \\
\hline Motile, one polar flagellum & $3(100)$ & 13 (87) [nonmotile: K-532, K-614] \\
\hline Accumulation of poly- $\beta$-hydroxybutyrate ${ }^{a}$ & $0(0)$ & 8 (53) $[63,65,297,299, \mathrm{~K}-311, \mathrm{~K}-379, \mathrm{~K}-532, \mathrm{~K}-614]$ \\
\hline Arginine dihydrolase & $3(100)$ & 11 (73) [all except $66,417, \mathrm{~K}-401, \mathrm{~K} 546$ ] \\
\hline Denitrification $^{b}$ & $3(100)$ & $6(40)[66,440, \mathrm{~K}-311, \mathrm{~K}-379, \mathrm{~K}-401, \mathrm{~K}-614]$ \\
\hline Pigmentation & $1(33)[\mathrm{K}-477]^{\mathrm{C}}$ & $0(0)$ \\
\hline Growth at $4^{\circ} \mathrm{C}$ & $0(0)$ & $0(0)$ \\
\hline Growth at $41^{\circ} \mathrm{C}$ & $3(100)$ & $15(100)$ \\
\hline \multicolumn{3}{|l|}{ Extracellular hydrolases } \\
\hline Gelatin $^{d}$ & $1(33)[142]$ & $2(13)[299,417]$ \\
\hline Starch & $0(0)$ & $0(0)$ \\
\hline Tween 80 & $1(33)[\mathrm{K}-477]$ & $0(0)$ \\
\hline Egg yolk & $1(33)[\mathrm{K}-477]$ & $0(0)$ \\
\hline Poly- $\beta$-hydroxybutyrate & $0(0)$ & $0(0)$ \\
\hline Autotrophic growth with hydrogen & $0(0)$ & $0(0)$ \\
\hline
\end{tabular}

a Most of the positive strains were not readily identifiable as accumulators on the basis of microscopic observation of granules but were characterized by the extraction and chemical identification of the polymer from the cells.

${ }^{b}$ With the exception of K-477, all positive strains exhibited anaerobic growth with nitrite accumulation but no gas production. Strain K-477 produced gas.

c Yellow colonies (carotenoid pigment with absorption peaks at 417 and $434 \mathrm{~nm}$ ).

${ }^{d}$ All positive reactions were weak.

TABLE 2. Utilization of substrates by strains of the "P. alcaligenes" group

\begin{tabular}{|c|c|c|}
\hline Class of compounds & $\begin{array}{l}\text { Compounds utilized } \\
\text { by all strains }\end{array}$ & Compounds not utilized by any strain \\
\hline Hydrocarbons & & $n$-Hexadecane \\
\hline $\begin{array}{l}\text { Carbohydrates and sugar } \\
\text { acids }\end{array}$ & & $\begin{array}{l}\text { D-Ribose, D-xylose, D-arabinose, L-arabinose, D-fucose, L-rhamnose, } \\
\text { glucose, mannose, galactose, sucrose, trehalose, maltose, cello- } \\
\text { biose, lactose, starch, salicin, 2-keto-gluconate, saccharate, mu- } \\
\text { cate }\end{array}$ \\
\hline Fatty acids & $\begin{array}{l}\text { Acetate, propionate, } \\
\text { caprate }\end{array}$ & Isobutyrate, isovalerate \\
\hline Dicarboxylic acids & Succinate, fumarate & Malonate, maleate, adipate, pimelate, suberate, azelate, sebacate \\
\hline Hydroxy acids & $\begin{array}{l}\text { Lactate, pyruvate, } \\
\alpha \text {-ketoglutarate }\end{array}$ & $\begin{array}{l}\text { Glycollate, D-(-)-tartrate, D- }(+) \text {-tartrate, } m \text {-tartrate, hydroxymeth- } \\
\text { ylglutarate }\end{array}$ \\
\hline Other organic acids & & Levulinate, citraconate \\
\hline Alcohols and polyols & & $\begin{array}{l}\text { Isopropanol, geraniol, 2,3-butylene glycol, erythritol, mannitol, } m \text { - } \\
\text { inositol, adonitol }\end{array}$ \\
\hline $\begin{array}{l}\text { Nonnitrogenous aromatic } \\
\text { and other cyclic com- } \\
\text { pounds }\end{array}$ & & $\begin{array}{l}\text { D-Mandelate, L-mandelate, benzoyl formate, } o \text {-hydroxybenzoate, } m \text { - } \\
\text { hydroxybenzoate, phthalate, phenylacetate, naphthalene, phenol, } \\
\text { quinate, testosterone }\end{array}$ \\
\hline $\begin{array}{l}\text { Amino acids and related } \\
\text { compounds }\end{array}$ & $\gamma$-Aminobutyrate & $\begin{array}{l}\text { L-Threonine, L-isoleucine, L-norleucine, L-valine, L-aspartate, L-ly- } \\
\text { sine, L-citrulline, } \alpha \text {-aminobutyrate, } \alpha \text {-aminovalerate, L-trypto- } \\
\text { phan, D-tryptophan, kyurenine, kyurenate, anthranilate }\end{array}$ \\
\hline
\end{tabular}


TABLE 3. Substrates used by some but not all strains of $P$. alcaligenes and $P$. pseudoalcaligenes

\begin{tabular}{|c|c|c|}
\hline \multirow{2}{*}{ Substrate } & \multicolumn{2}{|r|}{ No. $(\%)$ of positive strains } \\
\hline & $\begin{array}{l}P \text {. alcaligenes } \\
{[N=3]}\end{array}$ & $\begin{array}{c}P . \text { pseudoalcaligenes } \\
{[N=15]}\end{array}$ \\
\hline$n$-Dodecane & $1(33)[\mathrm{K}-477]$ & $0(0)$ \\
\hline Fructose & $0(0)$ & $15(100)$ \\
\hline Gluconate & $0(0)$ & 4 (27) [K-379, K-532, K-546, K-614] \\
\hline Butyrate & $2(67)[142, \mathrm{~K}-384]$ & 13 (87) [all except $440, \mathrm{~K}-504$ ] \\
\hline Valerate & $1(33)[142]$ & $6(40)[63,65,66,297,299, \mathrm{~K}-401]$ \\
\hline Heptanoate & $2(67)[\mathrm{K}-384, \mathrm{~K}-477]$ & 6 (40) $[440, \mathrm{~K}-311, \mathrm{~K}-379, \mathrm{~K}-532, \mathrm{~K}-546, \mathrm{~K}-614]$ \\
\hline Caproate & $1(33)[K-477]$ & $5(33)[63,65,299, \mathrm{~K}-379, \mathrm{~K}-546]$ \\
\hline Caprylate & $3(100)$ & 14 (93) [all except K-401] \\
\hline Pelargonate & $3(100)$ & 13 (87) [all except $\mathrm{K}-401, \mathrm{~K}-504$ ] \\
\hline Glutarate & $0(0)$ & $8(53)[63,65,66,297,299,417, \mathrm{~K}-532, \mathrm{~K}-614]$ \\
\hline Glycerate & $0(0)$ & 14 (93) [all except 66] \\
\hline D-Malate & $0(0)$ & 12 (80) [all except $440, \mathrm{~K}-311, \mathrm{~K}-546$ ] \\
\hline L-Malate & $3(100)$ & 14 (93) [all except K-311] \\
\hline$\beta$-Hydroxybutyrate & $0(0)$ & 14 (93) [all except K-546] \\
\hline Citrate & $2(67)[142, \mathrm{~K}-384]$ & $4(27)[63,65, \mathrm{~K}-532, \mathrm{~K}-614]$ \\
\hline Aconitate & 1 (33) $[\mathrm{K}-384]$ & 2 (13) [K-532, K-614] \\
\hline Itaconate & $0(0)$ & 11 (73) [all except 440, K-311, K-379, K-546] \\
\hline Mesaconate & $0(0)$ & 13 (87) [all except K-311, 440] \\
\hline Ethanol & $1(33)[K-477]$ & $15(100)$ \\
\hline$n$-Propanol & $1(33)[K-477]$ & $15(100)$ \\
\hline Butanol & $2(67)[\mathrm{K}-384, \mathrm{~K}-477]$ & $15(100)$ \\
\hline Isobutanol & $1(33)[K-477]$ & $0(0)$ \\
\hline Ethylene glycol & $0(0)$ & 4 (27) $[441, \mathrm{~K}-311, \mathrm{~K}-379, \mathrm{~K}-504]$ \\
\hline Propylene glycol & $1(33)[\mathrm{K}-477]$ & 13 (87) [all except $63, \mathrm{~K}-401$ ] \\
\hline Glycerol & $0(0)$ & $3(20)[297,299, \mathrm{~K}-401]$ \\
\hline Sorbitol & $0(0)$ & 4 (27) $[441, \mathrm{~K}-311, \mathrm{~K}-379, \mathrm{~K}-546]$ \\
\hline$p$-Hydroxybenzoate & $0(0)$ & $1(7)[440]$ \\
\hline Glycine & $0(0)$ & 7 (47) $[65,297,299,440, \mathrm{~K}-401, \mathrm{~K}-532, \mathrm{~K}-614]$ \\
\hline L-Alanine & $3(100)$ & 14 (93) [all except K-546] \\
\hline D-Alanine & $1(33)[K-477]$ & 10 (67) [all except 417, 441, K-379, K-504, K-546] \\
\hline$\beta$-Alanine & $2(67)[142, \mathrm{~K}-477]$ & 10 (67) [all except 440, K-311, K-379, K-401, K-504] \\
\hline L-Serine & $0(0)$ & 10 (67) [all except 441, K-311, K-504, K-532, K-546] \\
\hline L-Leucine & $3(100)$ & $6(40)[63,65,66,297,299,417]$ \\
\hline L-Glutamate & $3(100)$ & 13 (87) [all except $441, \mathrm{~K}-504]$ \\
\hline L-Arginine & $3(100)$ & 14 (93) [all except $\mathrm{K}-401]$ \\
\hline L-Ornithine & $0(0)$ & $1(7)[\mathrm{K}-311]$ \\
\hline$\delta$-Aminovalerate & $1(33)[142]$ & $7(47)[63,65,66,297,299,417,440]$ \\
\hline L-Histidine & $2(67)[142, \mathrm{~K}-384]$ & $6(40)[63,65,297,299, \mathrm{~K}-532, \mathrm{~K}-614]$ \\
\hline L-Proline & $3(100)$ & $13(87)$ [all except 66,417 ] \\
\hline L-Tyrosine & $1(33)[\mathrm{K}-384]$ & 10 (67) [all except $\mathrm{K}-379, \mathrm{~K}-401, \mathrm{~K}-504, \mathrm{~K}-532, \mathrm{~K}-614$ ] \\
\hline L-Phenylalanine & $0(0)$ & $6(40)[63,65,66,297,299,417]$ \\
\hline Histamine & $1(33)[\mathrm{K}-477]$ & $2(13)[\mathrm{K}-379, \mathrm{~K}-401]$ \\
\hline Ethanolamine & $0(0)$ & 13 (87) [all except 440,441$]$ \\
\hline Putrescine & $2(67)[\mathrm{K}-384, \mathrm{~K}-477]$ & 14 (93) [all except K-504] \\
\hline Spermine & $3(100)$ & $13(87)$ [all except $\mathrm{K}-401, \mathrm{~K}-504]$ \\
\hline Butylamine & $1(33)[\mathrm{K}-477]$ & $0(0)$ \\
\hline$\alpha$-Amylamine & $1(33)[\mathrm{K}-477]$ & $0(0)$ \\
\hline Betaine & $0(0)$ & $13(87)$ [all except 440,441$]$ \\
\hline Sarcosine & $0(0)$ & $8(53)[65,66,417,441, \mathrm{~K}-379, \mathrm{~K}-504, \mathrm{~K}-532, \mathrm{~K}-614]$ \\
\hline Creatine & $0(0)$ & $2(13)[\mathrm{K}-311, \mathrm{~K}-401]$ \\
\hline Trigonelline & $0(0)$ & $1(7)[\mathrm{K}-311]$ \\
\hline
\end{tabular}

ethanolamine, and betaine) were used by all but one or two of the $P$. pseudoalcaligenes strains examined.

The results of DNA-DNA hybridization experiments using the type strains $P$. alcaligenes 142 and $P$. pseudoalcaligenes 63 as references are presented in Tables 4 and 5 along with the DNA base compositions of all strains included in the study. The results of additional hybridization experiments using as reference strain $P$. pseudoalcaligenes $\mathrm{K}-546$, which differs from the type strain of the same species with respect 
TABLE 4. DNA-DNA hybridization data from selected Pseudomonas strains, using P. alcaligenes 142 as reference, compared with similarity $\left(S_{J}\right)$ and matching $\left(S_{S M}\right)$ coefficients

\begin{tabular}{|c|c|c|c|c|c|c|}
\hline \multirow{2}{*}{ DNA from: } & \multirow{2}{*}{$\begin{array}{l}\text { Mol\% } \\
\mathrm{G}+\mathrm{C}\end{array}$} & \multicolumn{2}{|c|}{ Competition (\%) at: } & \multirow{2}{*}{$\begin{array}{c}\text { Direct } \\
\text { binding } \\
(\%)\end{array}$} & \multirow{2}{*}{$\mathbf{S}_{\mathrm{J}}$} & \multirow{2}{*}{$\mathrm{S}_{\mathrm{SM}}$} \\
\hline & & $72^{\circ} \mathrm{C}$ & $80^{\circ} \mathrm{C}$ & & & \\
\hline$P$. alcaligenes 142 & 66 & $" 100 "$ & $" 100 "$ & $" 100 "$ & $" 100 "$ & "100" \\
\hline$P$. alcaligenes $\mathrm{K}-384$ & 66 & 76 & 81 & 46 & 53 & 88 \\
\hline$P$. alcaligenes $\mathrm{K}-477$ & 68 & 53 & 33 & $\mathrm{ND}^{a}$ & 51 & 87 \\
\hline P. pseudoalcaligenes 63 & 62 & 30 & 2 & 27 & 51 & 85 \\
\hline$P$. pseudoalcaligenes 66 & 63 & 18 & 0 & ND & 43 & 82 \\
\hline P. pseudoalcaligenes $\mathrm{K}-311$ & 63 & 17 & 0 & ND & 39 & 81 \\
\hline$P$. pseudoalcaligenes $\mathrm{K}-379$ & 63 & 25 & ND & ND & 35 & 81 \\
\hline P. pseudoalcaligenes $\mathrm{K}-401$ & 63 & 37 & 14 & ND & 37 & 80 \\
\hline$P$. pseudoalcaligenes $\mathrm{K}-504$ & 63 & 29 & ND & ND & 36 & 82 \\
\hline$P$. pseudoalcaligenes $\mathrm{K}-532$ & 64 & 37 & ND & ND & 47 & 84 \\
\hline$P$. pseudoalcaligenes $\mathrm{K}-546$ & 63 & 34 & 11 & 26 & 43 & 83 \\
\hline$P$. pseudoalcaligenes 440 & 62 & 17 & 0 & ND & 47 & 85 \\
\hline$P$. pseudoalcaligenes 441 & 63 & 26 & 0 & ND & 42 & 82 \\
\hline P. stutzeri 222 & 65 & 37 & 8 & 13 & 29 & 70 \\
\hline P. mendocina $\mathrm{CH} 20$ & 64 & 29 & 4 & 17 & 37 & 73 \\
\hline P. aeruginosa 131 & 68 & 35 & 0 & 16 & 32 & 64 \\
\hline$P$. fluorescens A 192 & 61 & 16 & 3 & ND & 31 & 64 \\
\hline$P$. fluorescens B 400 & 62 & 33 & ND & ND & 28 & 66 \\
\hline$P$. fluorescens C 18 & 61 & 13 & ND & ND & 30 & 64 \\
\hline$P$. fluorescens E 86 & 64 & 15 & 0 & ND & 31 & 63 \\
\hline$P$. putida A 7 & 62 & 23 & 14 & ND & $\begin{array}{l}01 \\
28\end{array}$ & 62 \\
\hline$P$. putida B 52 & 60 & 52 & ND & ND & 24 & 55 \\
\hline$P$ syringae 755 & 59 & 0 & ND & ND & 26 & 67 \\
\hline P. acidovorans 105 & 66 & 8 & ND & ND & 23 & 60 \\
\hline P. cepacia 382 & 67 & 0 & ND & ND & 24 & 47 \\
\hline
\end{tabular}

a ND, Not determined.

to many phenotypic traits, are presented in Table 6. Strain K-546, as reference for competition experiments, gave higher homology values than did strain 63 for all species tested, although the same relative relationships are indicated in each case. The reason for this discrepancy is not clear, but such problems with competition experiments are occasionally encountered (5). The two strains did, however, give comparable results in the direct-binding experiments.

In contrast to the phenotypic studies, the hybridization experiments indicate a relatively high internal homology within each of the two species and a clear separation between them. Both species appear to be closely related to species of the "stutzeri" group ( $P$. mendocina and $P$. stutzeri) and more distantly related to the "fluorescent pseudomonads." The close relationship of the "alcaligenes" group with $P$. mendocina is also reflected in some of the phenotypic data; strain K-477 was shown to differ from all the other strains of the group with respect to two characters which it shares with $P$. mendocina (carotenoid pigment production and denitrification with gas production). However, the overall similarity of this strain to the type strain of $P$. mendocina $\left(\mathrm{S}_{\mathrm{J}}=46, \mathrm{~S}_{\mathrm{SM}}=72\right)$ was less than its similarity with $P$. alcaligenes $142\left(\mathrm{~S}_{\mathrm{J}}=51, \mathrm{~S}_{\mathrm{SM}}=87\right)$.

The results of the hybridization experiments were surprising in light of the many previous studies which, taken together, had indicated that the ability to accumulate poly- $\beta$-hydroxybutyric acid (PHB) as a cellular reserve was a taxonomic character of particular importance within the genus Pseudomonas (1). Although many of the strains of the "alcaligenes" group were found to accumulate PHB, neither species showed homology with any of the other PHB. accumulating Pseudomonas species but were shown instead to be part of the large homology group (8) of otherwise PHB-negative Pseudomonas species.

The following description of the alcaligenes group serves to distinguish strains of $P$. alcali- 
genes and $P$. pseudoalcaligenes from other members of the genus Pseudomonas:

Motile by means of a single polar flagellum ${ }^{a}$ (with the exception of the nonmotile strains K-532 and K-614).

Soluble pigments not produced ${ }^{b}$

Oxidase reaction positive. ${ }^{c}$

Organic growth factors not required ${ }^{d}$

No hydrolysis of starch $^{e}$ or extracellular poly$\beta$-hydroxybutyrate. ${ }^{f}$

No autotrophic growth with the oxidation of $\mathrm{H}_{2}$ as energy source. ${ }^{g}$

Growth at $41^{\circ} \mathrm{C}^{h}$ but not at $4^{\circ} \mathrm{C}$. $^{i}$

Organic compounds which can be used as sole carbon sources: caprate,${ }^{j}$ lactate,${ }^{k}$ and $\gamma$ aminobutyrate. ${ }^{l}$

Organic compounds which cannot be used as sole carbon sources: glucose, ${ }^{m}$ cellobiose, ${ }^{n}$ malonate, ${ }^{o}$ sebacate ${ }^{p}$ glycolate,$^{q}$ isoleucine ${ }^{r}$ and aspartate. ${ }^{s}$

${ }^{a-s}$ Other Pseudomonas species differ from $P$. alcaligenes and $P$. pseudoalcaligenes with respect to the following traits: ${ }^{b, m, o, p, s} P$. aeruginosa; ${ }^{a, b, h, i, m, o, r, s} P$. fluorescens; ${ }^{a, b, h, m, o, r, s} P$. putida; ${ }^{a, b, c, h, m, o, s^{s} P} P$. syringae" groups; ${ }^{a, b, h, m, 0, s} P$. cichorii; ${ }^{m, o, q, r, s} P$. mendocina; ${ }^{e, m, o, p, q, r} P$. stutzeri; ${ }^{a, h, o, p, q, r, s} P$. acidovorans; ${ }^{a, h, j, l, p, q, r, s} P$. testosteroni; ${ }^{a, m, n, o, p, q, r, s} P$. cepacia; ${ }^{a, m, n, o, p, r, s} P$. mar-

TABLE 5. DNA-DNA hybridization data from selected Pseudomonas strains, using $P$. pseudoalcaligenes 63 as reference, compared with similarity $\left(S_{J}\right)$ and matching $\left(S_{S M}\right)$ coefficients

\begin{tabular}{|c|c|c|c|c|c|c|}
\hline \multirow{2}{*}{ DNA from: } & \multirow{2}{*}{$\begin{array}{l}\text { Mol\% } \\
\text { G+C }\end{array}$} & \multicolumn{2}{|c|}{ Competition (\%) at: } & \multirow{2}{*}{$\begin{array}{c}\text { Direct } \\
\text { binding } \\
(\%)\end{array}$} & \multirow{2}{*}{$\mathrm{S}_{\mathrm{J}}$} & \multirow{2}{*}{$\mathrm{S}_{\mathrm{SM}}$} \\
\hline & & $71^{\circ} \mathrm{C}$ & $80^{\circ} \mathrm{C}$ & & & \\
\hline P.pseudoalcaligenes 63 & 62 & $" 100 "$ & "100" & $" 100 "$ & $" 100 "$ & "100" \\
\hline P. pseudoalcaligenes 65 & 62 & 82 & 86 & $\mathrm{ND}^{a}$ & 92 & 98 \\
\hline P. pseudoalcaligenes 66 & 63 & 77 & 74 & ND & 82 & 94 \\
\hline P. pseudoalcaligenes 297 & 64 & 79 & ND & ND & 88 & 96 \\
\hline P. pseudoalcaligenes 299 & 62 & 79 & 71 & ND & 88 & 96 \\
\hline P. pseudoalcaligenes 417 & 63 & 79 & ND & ND & 77 & 93 \\
\hline P. pseudoalcaligenes 440 & 62 & 57 & 53 & ND & 58 & 87 \\
\hline P. pseudoalcaligenes 441 & 63 & 71 & 71 & ND & 55 & 84 \\
\hline P. pseudoalcaligenes $\mathrm{K}-311$ & 63 & 73 & 73 & ND & 53 & 83 \\
\hline P. pseudoalcaligenes K-379 & 63 & 76 & ND & ND & 53 & 83 \\
\hline P. pseudoalcaligenes K-401 & 63 & 79 & 82 & ND & 53 & 85 \\
\hline P. pseudoalcaligenes K-504 & 63 & 81 & ND & ND & 48 & 83 \\
\hline P. pseudoalcaligenes K-532 & 64 & 70 & ND & ND & 63 & 88 \\
\hline P. pseudoalcaligenes K-546 & 63 & 79 & 84 & ND & 56 & 85 \\
\hline P. pseudoalcaligenes K-614 & 63 & 63 & ND & ND & 63 & 88 \\
\hline P. alcaligenes 142 & 66 & 36 & 22 & 26 & 51 & 85 \\
\hline$P$. alcaligenes K-384 & 66 & 37 & 21 & ND & 52 & 84 \\
\hline$P$. alcaligenes $\mathrm{K}-477$ & 68 & 36 & 21 & ND & 45 & 81 \\
\hline P. stutzeri 222 & 65 & 20 & 9 & 15 & 46 & 76 \\
\hline P. mendocina $\mathrm{CH} 20$ & 64 & 51 & 42 & 36 & 56 & 80 \\
\hline P. aeruginosa 131 & 68 & 33 & 22 & 18 & 46 & 70 \\
\hline P. fluorescens A 192 & 61 & 18 & 17 & ND & 42 & 68 \\
\hline$P$. fluorescens A 12 & 61 & 25 & ND & ND & 43 & 71 \\
\hline P. fluorescens B 400 & 62 & 11 & ND & ND & 40 & 69 \\
\hline P. fluorescens C 18 & 61 & 15 & ND & ND & 39 & 67 \\
\hline P. fluorescens D 31 & 63 & 23 & ND & ND & 43 & 67 \\
\hline P. putida A 90 & 63 & 21 & 24 & ND & 43 & 69 \\
\hline P. putida B 53 & 60 & 31 & ND & ND & 37 & 62 \\
\hline P. syringae 755 & 59 & 12 & ND & ND & 39 & 71 \\
\hline P. acidovorans 105 & 66 & 3 & ND & ND & 38 & 67 \\
\hline P. cepacia 382 & 67 & 5 & ND & ND & 34 & 53 \\
\hline P. facilis 332 & 64 & 0 & ND & ND & 28 & 71 \\
\hline P. saccharophila & 69 & 0 & ND & ND & 24 & 65 \\
\hline
\end{tabular}

${ }^{a}$ ND, Not determined. 
TABLE 6. DNA-DNA hybridization from selected $P$ seudomonas strains, using $P$. pseudoalcaligenes $K$ 546 as reference, compared with similarity $\left(S_{J}\right)$ and matching $\left(S_{S M}\right)$ coefficients $^{a}$

\begin{tabular}{|c|c|c|c|c|}
\hline DNA from: & $\begin{array}{c}\text { Compe- } \\
\text { tition } \\
\text { at } 71^{\circ} \mathrm{C} \\
(\%)\end{array}$ & $\begin{array}{c}\text { Direct } \\
\text { binding } \\
(\%)\end{array}$ & $\mathrm{S}_{\mathrm{J}}$ & $\mathbf{S}_{\mathrm{SM}}$ \\
\hline P. pseudoalcaligenes K-546 & "100" & "100" & $" 100 "$ & $" 100 "$ \\
\hline$P$. pseudoalcaligenes 63 & 97 & 74 & 56 & 85 \\
\hline P. pseudoalcaligenes 65 & 88 & $\mathrm{ND}^{b}$ & 58 & 85 \\
\hline P. pseudoalcaligenes 66 & 83 & ND & 55 & 85 \\
\hline P. pseudoalcaligenes 297 & 90 & ND & 57 & 85 \\
\hline P. pseudoalcaligenes K-311 & 92 & ND & 69 & 91 \\
\hline$P$. pseudoalcaligenes $\mathrm{K}-401$ & 90 & ND & 48 & 83 \\
\hline$P$. pseudoalcaligenes K-504 & 89 & ND & 62 & 89 \\
\hline P. pseudoalcaligenes K-532 & 87 & ND & 58 & 87 \\
\hline$P$. pseudoalcaligenes 440 & 79 & ND & 59 & 88 \\
\hline P. pseudoalcaligenes 441 & 84 & ND & 69 & 90 \\
\hline$P$. alcaligenes 142 & 51 & 28 & 43 & 83 \\
\hline$P$. alcaligenes $\mathrm{K}-384$ & 59 & ND & 56 & 87 \\
\hline$P$. alcaligenes $\mathrm{K}-477$ & 49 & ND & 54 & 86 \\
\hline P. aeruginosa 131 & 43 & 17 & 41 & 67 \\
\hline P. stutzeri 222 & 39 & 18 & 44 & 76 \\
\hline P. mendocina $\mathrm{CH} 20$ & 75 & 33 & 44 & 74 \\
\hline P. acidovorans 105 & 15 & ND & 25 & 58 \\
\hline P. testosteroni 78 & 0 & ND & 27 & 65 \\
\hline P. marginata 704 & 0 & ND & 31 & 53 \\
\hline P. caryophylli 720 & 8 & ND & 29 & 65 \\
\hline P. solanocearum 769 & 0 & ND & 30 & 73 \\
\hline P. maltophilia 67 & 8 & ND & 20 & 71 \\
\hline
\end{tabular}

a The moles percent guanine plus cytosine of the following strains which do not appear in Tables 4 and 5 are: $P$. testosteroni $78,61 \%$; $P$. marginata $704,69 \%$; $P$. caryophylli $720,65 \%$; $P$. solanocearum $769,66 \%$; and $P$. maltophilia 67 , $67 \%$.

${ }^{\circ} \mathrm{ND}$, Not determined.

ginata; ${ }^{a, j, m, n, 0, q, s} P$. caryophylli; ${ }^{h, j, m, s} P$. solanacearum; ${ }^{m, o, p, q, s} P$. pickettii; ${ }^{f, j, m, n, o, s} P$. mallei; $a, e, f, m, n, p, r, s P$. pseudomallei; ${ }^{d, j, k, l, s} P$. diminuta; $d, h, j, k, l, m, n, s P$. vesicularis; ${ }^{a, c, d, j, l, m, n, o} P$. maltophilia; ${ }^{f, h, j, k, l P}$. lemoignei; ${ }^{f, h, j, l, m, o, s} P$. delafieldii; $f, g, h, j, l, m, o, s P$. facilis; ${ }^{e, g, h, j, l, m, n, s} P$. saccharophila; ${ }_{g, h, j, l, m, s} P$. ruhlandii; ${ }^{g, h, j, l, m, n, s} P$. flava; ${ }^{g, h, l, m, q, s, P}$. palleronii.

\section{ACKNOWLEDGMENT}

We thank Manley Mandel for determining the DNA composition of the new $P$. alcaligenes and $P$. pseudoalcaligenes strains.

This work was supported by Public Health Service grant AI-1808 from the National Institute of Allergy and Infectious Diseases.

\section{REPRINT REQUESTS}

Address reprint requests to: Dr. C. Ralston-Barrett, Department of Nutrition, University of California, Berkeley, CA 94720

\section{LITERATURE CITED}

1. Ballard, R. W., N. J. Palleroni, M. Doudoroff, R. Y. Stanier, and M. Mandel. 1970. Taxonomy of the aerobic pseudomonads: Pseudomonas cepacia, P. marginata, $P$. allicola, and $P$. caryophylli. J. Gen. Microbiol. 60:199-214.

2. Davis, D. H., R. Y. Stanier, M. Doudoroff, and $M$. Mandel. 1970. Taxonomic studies on some Gram negative polarly flagellated "hydrogen bacteria" and related species. Arch. Mikrobiol. 70:1-13.

3. Mandel, M. 1966. Deoxyribonucleic acid base composition in the genus Pseudomonas. J. Gen. Microbiol. 43:273-292.

4. Palleroni, N. J., R. W. Ballard, E. Ralston, and M. Doudoroff. 1972. Deoxyribonucleic acid homologies among some Pseudomonas species. J. Bacteriol. 110:1-11.

5. Palleroni, N. J., and M. Doudoroff. 1971. Phenotypic characterization and deoxyribonucleic acid homologies of Pseudomonas solanacearum. J. Bacteriol., 107:690-696.

6. Palleroni, N. J., and M. Doudoroff. 1972. Some properties and taxonomic subdivisions of the genus Pseudomonas. Annu. Rev. Phytopathol. 10:73-100.

7. Palleroni, N. J., M. Doudoroff, R. Y. Stanier, R. E. Solanes, and M. Mandel. 1970. Taxonomy of the aerobic pseudomonads: the properties of the Pseudomonas stutzeri group. J. Gen. Microbiol. 60:215-231.

8. Palleroni, N. J., R. Kunisawa, R. Contopoulou, and M. Doudoroff. 1973. Nucleic acid homologies in the genus Pseudomonas. Int. J. Syst. Bacteriol. 23:333-339.

9. Ralston, E., N. J. Palleroni, and M. Doudoroff. 1973. Pseudomonas pickettii, a new species of clinical origin related to Pseudomonas solanacearum. Int. J. Syst. Bacteriol. 23:15-19.

10. Sokal, R. R., and P. H. A. Sneath. 1963. Principles of numerical taxonomy. W. H. Freeman and Co., San Francisco.

11. Stanier, R. Y., N. J. Palleroni, and M. Doudoroff. 1966. The aerobic pseudomonads: a taxonomic study. J. Gen. Microbiol. 43:159-271. 